\title{
WEAKLY FINITELY PRESENTED INFINITE PERIODIC GROUPS
}

\author{
S. V. IVANOV
}

\begin{abstract}
A group $G$ given by a presentation $G=\langle\mathcal{A} \| \mathcal{R}\rangle$ is called weakly finitely presented if every finitely generated subgroup of $G$, generated by (images of) some words in $\mathcal{A}^{ \pm 1}$, is naturally isomorphic to the subgroup of a group $G_{0}=\left\langle\mathcal{A}_{0} \| \mathcal{R}_{0}\right\rangle$, where $\mathcal{A}_{0} \subseteq \mathcal{A}, \mathcal{R}_{0} \subseteq \mathcal{R}$ are finite, generated by (images of) the same words. In the article, weakly finitely presented periodic groups which are not locally finite are constructed.
\end{abstract}

\section{INTRODUCTION}

Let a group $G$ be given by a presentation

$$
G=\langle\mathcal{A} \| R=1, R \in \mathcal{R}\rangle,
$$

where $\mathcal{A}$ is an alphabet, $\mathcal{R}$ is a set of defining relators (which are reduced words in the alphabet $\mathcal{A}^{ \pm 1}=\mathcal{A} \cup \mathcal{A}^{-1}$ ). Let $F(\mathcal{A})$ be a free group in the alphabet $\mathcal{A}, N(\mathcal{R})$ the normal closure of $\mathcal{R}$ in $F(\mathcal{A})$ and

$$
\psi_{0}: F(\mathcal{A}) \rightarrow G=F(\mathcal{A}) / N(\mathcal{R})
$$

be the natural homomorphism.

This group presentation (1) is referred to as weakly finite if for every finite set $\mathcal{S}=$ $\left\{W_{1}, \ldots, W_{m}\right\}$ of words $W_{1}, \ldots, W_{m}$ in $\mathcal{A}^{ \pm 1}$, called $\mathcal{A}$-words, there are finite subsets $\mathcal{A}_{\mathcal{S}} \subseteq \mathcal{A}$ and $\mathcal{R}_{\mathcal{S}} \subseteq \mathcal{R}$ such that $W_{1}, \ldots, W_{m}$ are $\mathcal{A}_{\mathcal{S}}$-words and the subgroup $\left\langle\mathcal{S}^{\psi_{0}}\right\rangle$ of $G=F(\mathcal{A})^{\psi_{0}}$ generated by $\mathcal{S}^{\psi_{0}}$ is naturally isomorphic to the subgroup $\left\langle\mathcal{S} \psi_{0, \mathcal{S}}\right\rangle$ of the group $G_{\mathcal{S}}=F\left(\mathcal{A}_{\mathcal{S}}\right)^{\psi_{0}, \mathcal{S}}$, where

$$
\psi_{0, \mathcal{S}}: F\left(\mathcal{A}_{\mathcal{S}}\right) \rightarrow G_{\mathcal{S}}=F\left(\mathcal{A}_{\mathcal{S}}\right) / N\left(\mathcal{R}_{\mathcal{S}}\right)
$$

is the natural homomorphism. Note that, in the foregoing notation, there is always a natural homomorphism

$$
\left\langle\mathcal{S}^{\psi_{\mathcal{S}}}\right\rangle \rightarrow\left\langle\mathcal{S}^{\psi}\right\rangle
$$

for the natural homomorphism $\langle\mathcal{S}\rangle \rightarrow\left\langle\mathcal{S}^{\psi}\right\rangle$ factors out through $\left\langle\mathcal{S}^{\psi_{\mathcal{S}}}\right\rangle$.

Accordingly, a group $G$ given by (1) is called weakly finitely presented if (1) is a weakly finite presentation.

Recall that the presentation (1) is called finite if both $\mathcal{A}$ and $\mathcal{R}$ are finite. It is easy to see that if $\mathcal{A}$ is finite then (1) is weakly finite if and only if there is a finite subset $\mathcal{R}_{0} \subseteq \mathcal{R}$ such that $N\left(\mathcal{R}_{0}\right)=N(\mathcal{R})$. It is also easy to see that the presentation (1) is weakly finite if every finitely generated subgroup of $G$ is finitely presentable (that is, has a finite presentation). In particular, if $G$ is a locally finite group then $G$ is weakly finitely presented.

2000 Mathematics Subject Classification. Primary 20E07, 20F05, 20F06, 20 F50.

Supported in part by NSF grants DMS 98-01500, DMS 00-99612. 
It seems rather natural to ask whether every periodic weakly finitely presented group is locally finite. This problem can be regarded as a weakened version of a long-standing problem, attributed to P.S. Novikov, on the existence of a finitely presented infinite periodic group. In this article, we will solve the former problem in the negative thus making some progress towards the Novikov problem. However, our construction of weakly finitely presented non-locally finite periodic groups does not even allow to bound the orders of elements and the problem on the existence of weakly finitely presented groups of bounded exponent that are not locally finite might be worth further investigation.

Recall that first examples of finitely generated infinite periodic groups of unbounded exponent were constructed by Golod eG64. Later more examples of such groups (which are rather easy to construct) were found by Aleshin [svA72], Grigorchuk rG80, Gupta and Sidki GS83, nG89]. Also, recall that the Burnside problem [B02 on periodic groups asks about the existence of finitely generated groups of exponent $n$ (that is, satisfying the identity $x^{n} \equiv 1$ ). For odd exponents $n \gg 1$ the Burnside problem was solved by Novikov and Adian [NA68 in 1968 (a simpler geometric solution for odd $n \gg 1$ was later found by Ol'shanskii's O82, O89|). The case of even exponents in the Burnside problem turned out to be much more difficult and required creation of new heavy machinery, see author's article [94] (or Lysenok's article [L96]), that was aimed to handle noncyclic centralizers (more applications of this machinery can be found in [IO96], [IO97], [100]). Curiously, many parts of the machinery of article [94] will be "recycled" in this paper in order to construct weakly finitely presented non-locally finite periodic groups whose inductive construction also generates noncyclic centralizers.

Recall that an $m$-generator free Burnside group $B(m, n)$ of exponent $n$ is the quotient $F_{m} / F_{m}^{n}$, where $F_{m}$ is a free group of rank $m$ and $F_{m}^{n}$ is the subgroup of $F_{m}$ generated by all $n$th powers of elements of $F_{m}$. By Novikov-Adian theorem (see NA68], [siA75]), $B(m, n)$ is infinite if $m>1$ and $n$ is odd, $n \geq 665$ (see also [082, O89, [194]).

Theorem A. Suppose that $N_{0}>2^{16}$ is an odd integer. Then there is a weakly finitely presented periodic group

$$
G=\langle\mathcal{A} \| R=1, R \in \mathcal{R}\rangle
$$

where both $\mathcal{A}$ and $\mathcal{R}$ are countably infinite, such that $G$ has unbounded exponent and $G$ is not a locally finite group for $G$ contains a subgroup isomorphic to a 2-generator free Burnside group $B\left(2, N_{0}\right)$ of exponent $N_{0}$.

A detailed description of our construction of weakly finitely presented periodic groups is given in the following.

Theorem B. Suppose that $N_{0}>2^{16}$ is an odd integer,

$$
G_{0}=\left\langle\mathcal{A}_{0} \| R=1, R \in \mathcal{R}_{0}\right\rangle
$$

is a finitely presented group and the alphabet $\mathcal{A}_{0}$ contains at least two letters. Then one can construct finite alphabets

$$
\mathcal{A}_{0} \subset \mathcal{A}_{1} \subset \mathcal{A}_{2} \subset \ldots
$$

and finite sets

$$
\mathcal{R}_{0} \subset \mathcal{R}_{1} \subset \mathcal{R}_{2} \subset \ldots
$$

of words in $\mathcal{A}_{0}^{ \pm 1}, \mathcal{A}_{1}^{ \pm 1}, \mathcal{A}_{2}^{ \pm 1}, \ldots$, respectively, such that the following hold. 
Let

$$
G_{j}=\left\langle\mathcal{A}_{j} \| R=1, R \in \mathcal{R}_{j}\right\rangle
$$

$F\left(\mathcal{A}_{j}\right)$ be a free group in the alphabet $\mathcal{A}_{j}$ and

$$
\psi_{0, j}: F\left(\mathcal{A}_{j}\right) \rightarrow G_{j}
$$

be the natural homomorphism, $j=0,1,2, \ldots$ Then for all $j \geq 0, k \geq 1$ it is true that

$$
F\left(\mathcal{A}_{j}\right)^{\psi_{0, j+k}}=F\left(\mathcal{A}_{j}\right)^{\psi_{0, j}} /\left(F\left(\mathcal{A}_{j}\right)^{\psi_{0, j}}\right)^{N_{0}^{3^{j}}}=G_{j} / G_{j}^{N_{0}^{3^{j}}} .
$$

In particular, the group

$$
G_{\infty}=\left\langle\mathcal{A}_{\infty}=\bigcup_{j=0}^{\infty} \mathcal{A}_{j} \| R=1, R \in \mathcal{R}_{\infty}=\bigcup_{j=0}^{\infty} \mathcal{R}_{j}\right\rangle
$$

contains isomorphic copies of quotients

$$
G_{0} / G_{0}^{N_{0}}, G_{1} / G_{1}^{N_{0}^{3}}, G_{2} / G_{2}^{N_{0}^{3^{2}}}, \ldots, G_{j} / G_{j}^{N_{0}^{3^{j}}}, \ldots
$$

which, except possibly for $G_{0} / G_{0}^{N_{0}^{3}}$, are not locally finite and if $W_{1}, \ldots, W_{m}$ are words in $\mathcal{A}_{j}^{ \pm 1}$ and

$$
\psi_{0, \infty}: F\left(\mathcal{A}_{\infty}\right) \rightarrow G_{\infty}
$$

is the natural homomorphism then the subgroup $\left\langle W_{1}^{\psi_{0, \infty}}, \ldots, W_{m}^{\psi_{0, \infty}}\right\rangle$ of $G_{\infty}$ has exponent $N_{0}^{3^{j+1}}$ (i.e., it satisfies the identity $x^{N_{0}^{3^{j+1}}} \equiv 1$ ) and naturally isomorphic to

$$
\left\langle W_{1}^{\psi_{0, j+1}}, \ldots, W_{m}^{\psi_{0, j+1}}\right\rangle \subseteq G_{j+1}
$$

\section{The Main Construction}

Let $\mathcal{A}=\left\{a_{i} \mid i \in I\right\}$, where $I$ is a (finite) index set with $|I|>1$, be an alphabet. We also consider alphabets

$$
\mathcal{B}=\left\{b_{i} \mid i \in I\right\}, \quad \mathcal{X}=\left\{x_{i} \mid i \in I\right\}, \text { and }\{c, y\}
$$

such that $\mathcal{A}, \mathcal{B}, \mathcal{X},\{c, y\}$ are pairwise disjoint.

Suppose that $\mathcal{R}$ is a set of words in $\mathcal{A}^{ \pm 1}=\mathcal{A} \cup \mathcal{A}^{-1}$ and

$$
H=\langle\mathcal{A} \| R=1, R \in \mathcal{R}\rangle
$$

is a group presentation whose set of generators is $\mathcal{A}$ and whose set of defining relators is $\mathcal{R}$. Consider the following defining relations

$$
\begin{gathered}
x_{i} c x_{i}^{-1}=c b_{i}, \quad i \in I, \\
y b_{i} y^{-1}=b_{i} a_{i}, \quad i \in I, \\
c^{n}=1, \\
x_{i} b_{j}=b_{j} x_{i}, \quad i, j \in I, \\
a_{i} b_{j}=b_{j} a_{i}, \quad i, j \in I, \\
a_{i} c=c a_{i}, \quad i \in I, \\
y c=c y, \quad i \in I .
\end{gathered}
$$

Let a group $G$ be given by a presentation whose alphabet is

$$
\mathcal{U}=\mathcal{A} \cup \mathcal{B} \cup \mathcal{X} \cup\{c, y\}
$$


and whose set of defining relations consists of defining relations of (2) and relations (3)-(9), thus

$$
G=\left\langle a_{i}, b_{i}, x_{i}, c, y, i \in I \| R=1, R \in \mathcal{R},(3)-(9)\right\rangle .
$$

Denote a free group in the alphabet $\mathcal{A}$ by $F(\mathcal{A})$ and let

$$
\alpha_{0}: F(\mathcal{A}) \rightarrow H, \quad \beta_{0}: F(\mathcal{U}) \rightarrow G
$$

be natural homomorphisms.

A word $W$ in the alphabet $\mathcal{A}^{ \pm 1}$ is called an $\mathcal{A}$-word and denoted by $W=W(\mathcal{A})$. A substitution $b_{i} \rightarrow a_{i}, i \in I$, turns an $\mathcal{A}$-word $W(\mathcal{A})$ into a $\mathcal{B}$-word denoted by $W(\mathcal{B})$.

Lemma A. The map $a_{i}^{\alpha_{0}} \rightarrow a_{i}^{\beta_{0}}, i \in I$, extends to a homomorphism

$$
\psi_{1}: H \rightarrow G
$$

whose kernel Ker $\psi_{1}$ is $H^{n}=\left\langle h^{n} \mid h \in H\right\rangle$.

Proof. First we will show that $H^{n} \subseteq \operatorname{Ker} \psi_{1}$. Let $W=W(\mathcal{A})$ be an $\mathcal{A}$-word. According to relations (5), (3), (6), we have in the group $G$ the following equalities

$$
1 \stackrel{G}{=}\left(W(\mathcal{X}) c W(\mathcal{X})^{-1}\right)^{n} \stackrel{\underline{G}}{=}(c W(\mathcal{B}))^{n} .
$$

Conjugating the last word by $y$ and using relations (4), (7), (9), we further have

$$
1 \stackrel{G}{=}(c W(\mathcal{B}))^{n} \stackrel{G}{=}\left(y c W(\mathcal{B}) y^{-1}\right)^{n} \stackrel{G}{=}(c W(\mathcal{B}) W(\mathcal{A}))^{n} .
$$

Making use of relations (7)-(8), we can see that

$$
1 \stackrel{G}{=}(c W(\mathcal{B}))^{n} W(\mathcal{A})^{n},
$$

whence $W(\mathcal{A})^{n} \stackrel{G}{=} 1$. The inclusion $H^{n} \subseteq \operatorname{Ker} \psi_{1}$ is proven.

To prove the converse, consider the following presentation for

$$
K=H / H^{n}=\left\langle a_{i}, i \in I \| R=1, R \in \mathcal{R}, W^{n}=1, W \in F(\mathcal{A})\right\rangle
$$

and let

$$
\gamma_{0}: F(\mathcal{A}) \rightarrow K
$$

be the natural homomorphism.

Let

$$
B(\mathcal{B} \cup c, n)=F(\mathcal{B} \cup c) / F(\mathcal{B} \cup c)^{n}
$$

be a free Burnside group of exponent $n$ in the alphabet $\mathcal{B} \cup c$ and

$$
\delta_{0}: F(\mathcal{B} \cup c) \rightarrow B(\mathcal{B} \cup c, n)
$$

be the natural homomorphism. Consider the direct product

$$
P=B(\mathcal{B} \cup c, n) \times K
$$

Note that the map

$$
c^{\delta_{0}} \rightarrow c^{\delta_{0}} b_{i}^{\delta_{0}}, \quad b_{j}^{\delta_{0}} \rightarrow b_{j}^{\delta_{0}}, j \in I,
$$

extends to an automorphism of the Burnside group $B(\mathcal{B} \cup c, n)$. Hence we can consider an HNN-extension of $P$ with, say, stable letter $x_{i}$ given by the following relative presentation

$$
\left\langle P, x_{i} \| x_{i} c^{\delta_{0}} x_{i}^{-1}=c^{\delta_{0}} b_{i}^{\delta_{0}}, x_{i} b_{j}^{\delta_{0}} x_{i}^{-1}=b_{j}^{\delta_{0}}, j \in I\right\rangle .
$$


Also note that the map

$$
c^{\delta_{0}} \rightarrow c^{\delta_{0}}, \quad b_{j}^{\delta_{0}} \rightarrow b_{j}^{\delta_{0}} a_{j}^{\gamma_{0}}, j \in I,
$$

extends to an isomorphism of $B(\mathcal{B} \cup c, n)$ to a subgroup of $P$ generated by $c^{\delta_{0}}, b_{j}^{\delta_{0}} a_{j}^{\gamma_{0}}$, $j \in I$, because $K^{n}=\{1\}$ and $B(\mathcal{B} \cup c, n)$ is a free Burnside group of exponent $n$ with free generators $c^{\delta_{0}}, b_{j}^{\delta_{0}}, j \in I$.

Therefore, we can consider a multiple HNN-extension $E$ of $P$ with stable letters $y, x_{i}, i \in I$, defined by the following relative presentation

$$
\begin{gathered}
E=\left\langle P, y, x_{i}, i \in I \| x_{i} c^{\delta_{0}} x_{i}^{-1}=c^{\delta_{0}} b_{i}^{\delta_{0}}, x_{i} b_{j}^{\delta_{0}} x_{i}^{-1}=b_{j}^{\delta_{0}},\right. \\
\left.y c^{\delta_{0}} y^{-1}=c^{\delta_{0}}, y b_{k}^{\delta_{0}} y^{-1}=b_{k}^{\delta_{0}} a_{k}^{\gamma_{0}}, \quad i, j, k \in I\right\rangle .
\end{gathered}
$$

It remains to notice that the natural homomorphism $F(\mathcal{U}) \rightarrow E$ factors out through $G$ and $K=F(\mathcal{A})^{\gamma_{0}}$ naturally embeds in $E$. Therefore, the converse inclusion $\operatorname{Ker} \psi_{1} \subseteq H^{n}$ also holds and Lemma $\mathrm{A}$ is proven.

Consider the quotient $G / G^{n^{3}}$ and let

$$
\varepsilon_{0}: F(\mathcal{U}) \rightarrow G / G^{n^{3}}
$$

denote the natural homomorphism.

Lemma B. The map $a_{i}^{\beta_{0}} \rightarrow a_{i}^{\varepsilon_{0}}, i \in I$, extends to a monomorphism

$$
\varphi_{0}: F(\mathcal{A})^{\beta_{0}} \rightarrow F(\mathcal{U})^{\varepsilon_{0}}=G / G^{n^{3}} .
$$

Proof. Clearly, the map $a_{i}^{\beta_{0}} \rightarrow a_{i}^{\varepsilon_{0}}, i \in I$, extends to a homomorphism

$$
\varphi_{0}: F(\mathcal{A})^{\beta_{0}} \rightarrow F(\mathcal{U})^{\varepsilon_{0}}
$$

and we have to show that $\operatorname{Ker} \varphi_{0}=\{1\}$. To do this we first repeat the construction of the proof of Lemma A and define as there groups

$$
K, \quad B(\mathcal{B} \cup c, n), \quad P
$$

and natural homomorphisms

$$
\gamma_{0}: F(\mathcal{A}) \rightarrow K=H / H^{n}, \quad \delta_{0}: F(\mathcal{B} \cup c) \rightarrow B(\mathcal{B} \cup c, n) .
$$

We also consider a free Burnside group $B(\mathcal{X}, n)$ in the alphabet $\mathcal{X}=\left\{x_{i} \mid i \in I\right\}$ of exponent $n$ and let

$$
\omega_{0}: F(\mathcal{X}) \rightarrow B(\mathcal{X}, n)
$$

be the natural homomorphism. Note that the map

$$
c^{\delta_{0}} \rightarrow c^{\delta_{0}} b_{i}^{\delta_{0}}, b_{j}^{\delta_{0}} \rightarrow b_{j}^{\delta_{0}}, a_{k}^{\gamma_{0}} \rightarrow a_{k}^{\gamma_{0}}, \quad j, k \in I,
$$

extends to an automorphism $\zeta_{i}, i \in I$, of the direct product

$$
P=B(\mathcal{B} \cup c, n) \times K .
$$

Moreover, it is easy to see that the subgroup $\left\langle\zeta_{i} \mid i \in I\right\rangle \subseteq \operatorname{Aut} P$ is isomorphic to a free Burnside group of exponent $n$ which is freely generated by $\zeta_{i}, i \in I$. Hence, we can consider a semidirect product

$$
Q=P \lambda B(\mathcal{X}, n)
$$

of $P$ and $B(\mathcal{X}, n)$ so that if $p \in P$ then

$$
x_{i}^{\omega_{0}} p x_{i}^{-\omega_{0}}=\zeta_{i}(p) .
$$

Clearly, the group $P$ has exponent $n$ and so the group $Q$ has exponent $n^{2}$. 
Recall that, when proving Lemma A, we saw that the map

$$
\varkappa_{0}: c^{\delta_{0}} \rightarrow c^{\delta_{0}}, b_{i}^{\delta_{0}} \rightarrow b_{i}^{\delta_{0}} a_{i}^{\gamma_{0}}, i \in I,
$$

extends to an isomorphism

$$
\varkappa_{0}:\left\langle b_{i}^{\delta_{0}}, c^{\delta_{0}} \mid i \in I\right\rangle \rightarrow\left\langle b_{i}^{\delta_{0}} a_{i}^{\gamma_{0}}, c^{\delta_{0}} \mid i \in I\right\rangle
$$

of corresponding subgroups of $P$. Therefore, we can consider the following HNNextension of $Q$ with stable letter $y$

$$
\mathcal{G}=\left\langle Q, y \| y c^{\delta_{0}} y^{-1}=c^{\delta_{0}}, y b_{i}^{\delta_{0}} y^{-1}=b_{i}^{\delta_{0}} a_{i}^{\gamma_{0}}, i \in I\right\rangle .
$$

The proof of Lemma B will be continued in Sect. 3.

\section{Adjusting the Machinery of [94}

Now we will make use of the machinery of [94 to show (see Lemma C) that if $n>2^{16}$ is odd then the quotient $\mathcal{G} / \mathcal{G}^{n^{3}}$ of the group $\mathcal{G}$ defined by presentation (13) is infinite and $Q$ naturally embeds in $\mathcal{G} / \mathcal{G}^{n^{3}}$. Since the natural homomorphism

$$
K=H / H^{n} \rightarrow Q \rightarrow G / G^{n^{3}}
$$

(naturally) factors out through the quotient $\mathcal{G} / \mathcal{G}^{n^{3}}$, Lemma B will be proven.

Diagrams over the group $\mathcal{G}(0)=\mathcal{G}$ given by presentation (13) (or, briefly, over $\mathcal{G}$ ), called diagrams of rank 0 , are defined to be maps that have two types of 2-cells.

A 2-cell $\Pi$ of the first type, called a 0-square, has four edges in its counterclockwise oriented boundary (called contour)

$$
\partial \Pi=e_{1} e_{2} e_{3} e_{4}
$$

and

$$
\varphi\left(e_{1}\right)=\varphi\left(e_{3}\right)^{-1}=y, \quad \varphi\left(e_{2}\right)=g, \quad \varphi\left(e_{4}\right)=\varkappa_{0}(g)^{-1},
$$

where, as in [94], $\varphi$ is the labeling function, $g \in\left\langle b_{i}^{\delta_{0}}, c^{\delta_{0}} \mid i \in I\right\rangle \subseteq Q$ (perhaps, $g=1$ ) and $\varkappa_{0}$ is defined by (12). Observe that we use Greek letters with no indices exactly as in [994] (in particular, see table (2.4) in [94]).

A 2 -cell $\Pi^{\prime}$ of the second type, called a 0 -circle, has $\ell \geq 2$ edges in its contour $\partial \Pi^{\prime}=e_{1} \ldots e_{\ell}$ so that $\varphi\left(e_{1}\right), \ldots, \varphi\left(e_{\ell}\right) \in Q$ and the word

$$
\varphi\left(\partial \Pi^{\prime}\right)=\varphi\left(e_{1}\right) \ldots \varphi\left(e_{\ell}\right)
$$

equals 1 in $Q$.

Note that this definition of 2-cells in a diagram of rank 0 is a generalization of corresponding definitions in Ol'shanskii's book $\mathrm{O} 89$. According to the presentation (13), our basic alphabet is $Q_{y}=\{Q, y\}$ and, from now on (unless stated otherwise), all words will be those in the alphabet $Q_{y}^{ \pm 1}=\left\{Q, y, y^{-1}\right\}$, called $Q_{y}$-words.

Let

$$
U_{1}=S_{0,1} y^{\varepsilon_{1,1}} S_{1,1} \ldots y^{\varepsilon_{\ell_{1}, 1}} S_{\ell_{1}, 1}, \quad U_{2}=S_{0,2} y^{\varepsilon_{1,2}} S_{1,2} \ldots y^{\varepsilon_{\ell_{2}, 2}} S_{\ell_{2}, 2},
$$

where $\varepsilon_{1,1}, \ldots, \varepsilon_{\ell_{1}, 1}, \varepsilon_{1,2}, \ldots, \varepsilon_{\ell_{2}, 2} \in\{ \pm 1\}, S_{0,1}, \ldots, S_{\ell_{1}, 1}$ are $Q$-syllables of the word $U_{1}$ (that is, maximal subwords of $U_{1}$ all of whose letters are in $Q$; if $S_{0,1}$ is, in fact, missing in $U_{1}$, then we assume that $S_{0,1}=1 \in Q$ and set $S_{\ell_{1}, 1}=1$ if $S_{\ell_{1}, 1}$ is not present in $U_{1}$ ) and $S_{0,2}, \ldots, S_{\ell_{2}, 2}$ are $Q$-syllables of the word $U_{2}$.

We will write $U_{1}=U_{2}$ if $\ell_{1}=\ell_{2}, \varepsilon_{j, 1}=\varepsilon_{j, 2}$ for all $j=1, \ldots, \ell_{1}$, and $S_{j, 1}=S_{j, 2}$ in the group $Q$ for all $j=0, \ldots, \ell_{1}$. 
The length $\left|U_{1}\right|$ of a word $U_{1}$ is $\ell_{1}$, that is, the number of occurrences of $y^{ \pm 1}$ in $U_{1}$.

If the (images of) words $U_{1}, U_{2}$ are equal in the group $\mathcal{G}(0)=\mathcal{G}$ given by $(13)$ then we will write $U_{1} \stackrel{0}{=} U_{2}$.

By induction on $i \geq 0$ we will construct groups $\mathcal{G}(i)$. Assume that the group $\mathcal{G}(i), i \geq 0$, is already constructed as a quotient of the group of $\mathcal{G}(0)$ by means of defining relations. Define $\mathcal{X}_{i+1}$ to be a maximal set of all $Q_{y}$-words of length $i+1$ (if any) with respect to the following three properties.

(AB1) Every word $A \in \mathcal{X}_{i+1}$ begins with $y$ or $y^{-1}$.

(AB2) The image of every word $A \in \mathcal{X}_{i+1}$ has infinite order in the group $\mathcal{G}(i)$.

(AB3) If $A, B$ are distinct elements of $\mathcal{X}_{i+1}$ then the image of $A^{n^{2}}$ is not conjugate in $\mathcal{G}(i)$ to the image of $B^{n^{2}}$ or $B^{-n^{2}}$.

Note that it follows from the analogue of Lemma 18.2 in rank $i \geq 0$ that the set $\mathcal{X}_{i+1}$ is nonempty.

Similar to [O82, O89, [194, we will call a word $A \in \mathcal{X}_{i+1}$ a period of rank $i+1$.

Now we define the group $\mathcal{G}(i+1)$ by imposing all relations $A^{n^{3}}=1$, called relations of rank $i+1$, on the group $\mathcal{G}(i)$ :

$$
\mathcal{G}(i+1)=\left\langle\mathcal{G}(i) \| A^{n^{3}}=1, A \in \mathcal{X}_{i+1}\right\rangle .
$$

It is clear that

$$
\mathcal{G}(i+1)=\left\langle\mathcal{G} \| A^{n^{3}}=1, A \in \bigcup_{j=1}^{i+1} \mathcal{X}_{j}\right\rangle
$$

We also define the limit group $\mathcal{G}(\infty)$ by imposing on the free product $Q *\langle y\rangle_{\infty}$ all relations of all ranks $i=0,1,2, \ldots$ :

$$
\begin{aligned}
\mathcal{G}(\infty)=\left\langle Q, y \| y c^{\delta_{0}} y^{-1}=c^{\delta_{0}}, y b_{k}^{\delta_{0}} y^{-1}=b_{k}^{\delta_{0}} a_{k}^{\delta_{0}},\right. & k \in I, \\
& \left.A^{n^{3}}=1, A \in \bigcup_{j=1}^{\infty} \mathcal{X}_{j}\right\rangle .
\end{aligned}
$$

The main technical result relating to the group $\mathcal{G}(\infty)$ is the following (as was pointed out above, Lemma B is immediate from Lemma C).

Lemma C. Suppose that $n>2^{16}$ is odd. Then the group $\mathcal{G}(\infty)$ given by the presentation (14) has exponent $n^{3}$ and the group $Q$ naturally embeds in $\mathcal{G}(\infty)$.

Proof. We will make use of the machinery of the article [94 to prove Lemma C (cf. [00). In order to do that we will have to make necessary changes in definitions, statements of lemmas of I94 and their proofs.

First of all, we take into account that there are multiple periods of rank $i$ and the symbol $A_{i}$ will denote one of many periods of rank $i$ (note that the length $\left|A_{i}\right|$ of $A_{i}$ is now $i$ ). Next, the number $n$ in [I94] is replaced by $n^{3}$.

In the definition of an $A$-periodic word, it is now assumed that $A$ starts with $y$ or $y^{-1}$ and $A$ is not conjugate in $\Gamma$ to a power $B^{\ell}$ with $|B|<|A|$.

In addition to cells of positive rank, we also have (as in O89, IO96) cells of rank 0 (which are now either 0 -squares or 0 -circles). The equality $r(\Delta)=0$ now means that all cells in $\Delta$ have rank 0 .

If

$$
p=e_{1} \ldots e_{\ell},
$$


where $e_{1}, \ldots, e_{\ell}$ are edges, is a path in a diagram $\Delta$ of rank $i$ (that is, a diagram over the group $\mathcal{G}(i))$ then the $y$-length $|p|$ of $p$ is $|\varphi(p)|$, that is, $|p|$ is the number of edges of $p$ labeled by $y^{ \pm 1}$. The (strict) length of $p$, that is, the total number of edges of $p$, is $\ell$ and denoted by $\|p\|$.

In the definition (A1)-(A2) of $j$-compatibility (p.13 [94) we eliminate the part (A2) because $n^{3}$ is odd and, similar to I94 in the case when $n$ is odd, it will be proven in a new version of Sect. 19 [94 that there are no $\mathcal{F}\left(A_{j}\right)$-involutions and there is no $j$-compatibility of type (A2).

We can also drop the definition of self-compatible cells (p.13 [I94]) because they do not exist when $n^{3}$ is odd (which is again analogous to [194 in the case when $n$ is odd). Thus all lemmas in [194 whose conclusions deal with self-compatible cells, compatibility of type (A2) actually claim that their assumptions are false (e.g., see lemmas of Sect. 12 [194]). On the other hand, the existence of self-compatible cells in assumptions of lemmas of [194] is now understood as the existence of noncontractible $y$-annuli which we are about to define.

A $y$-annulus is defined to be an annular subdiagram $\Gamma$ in a diagram $\Delta$ of rank $i$ such that $\Gamma$ consists of 0 -squares $S_{1}, \ldots, S_{k}$ so that if

$$
\partial S_{\ell}=f_{1, \ell} e_{1, \ell} f_{2, \ell} e_{2, \ell},
$$

where $e_{1, \ell}, e_{2, \ell}$ are $y$-edges (that is, labeled by $y^{ \pm 1}$ ) of $\partial S_{\ell}, 1 \leq \ell \leq k$, then $e_{2, \ell}=e_{1, \ell+1}^{-1}$, where the second subscript is $\bmod k, \ell=1, \ldots, k$. If $\Gamma$ is contractible into a point in $\Delta$ then we will call $\Gamma$ a contractible $y$-annulus. Otherwise, $\Gamma$ is a noncontractible $y$-annulus. If $\Gamma$ is contractible in $\Delta$ and bounds a simply connected subdiagram $\Gamma_{0}$ in $\Delta$ with $\varphi\left(\partial \Gamma_{0}\right)=1$ in $Q$ then $\Gamma$ is termed a reducible $y$-annulus.

In the definition of a reduced (simply connected or not) diagram $\Delta$ of rank $i$ (p.13 [94), we additionally require that $\Delta$ contain no reducible $y$-annuli.

As in [194, we can always remove reducible pairs and reducible $y$-annuli in a diagram $\Delta$ of rank $i$ to obtain from $\Delta$ a reduced diagram $\Delta^{\prime}$ of rank $i$. Note that in general it is not possible to get rid of noncontractible $y$-annuli (in non-simply connected diagrams of rank $i$ ).

In the definition of a 0-bond $\mathrm{E}$ between $p$ and $q$ (p.15 [94]) we now require that E consist of several 0 -squares $S_{1}, \ldots, S_{k}$ so that if

$$
\partial S_{\ell}=f_{1, \ell} e_{1, \ell} f_{2, \ell} e_{2, \ell},
$$

where $e_{1, \ell}, e_{2, \ell}$ are $y$-edges of $\partial S_{\ell}, 1 \leq \ell \leq k$, then $e_{1,1}^{-1} \in p, e_{2, \ell}=e_{1, \ell+1}^{-1}$, $\ell=$ $1, \ldots, k-1$, and $e_{2, k}^{-1} \in q$.

The standard contour of the 0 -bond E between $p$ and $q$ is

$$
\partial \mathrm{E}=e_{1,1}^{-1}\left(f_{1,1}^{-1} \ldots f_{1, k}^{-1}\right) e_{2, k}^{-1}\left(f_{2, k}^{-1} \ldots f_{2,1}^{-1}\right)
$$

and the edges $e_{1,1}^{-1}, e_{2, k}^{-1}$ are denoted by $\mathrm{E} \wedge p, \mathrm{E} \wedge q$, respectively.

In the definition of a simple in rank $i$ word $A$ (p.19 [I94), we additionally require that $|A|>0$ and $A$ start with $y^{ \pm 1}$. Observe that it follows from Lemma 18.2 (in rank $i-1$ ) and definitions that a period of rank $i$ is simple in rank $i-1$ (and hence in any rank $j \leq i-1)$.

In the definition of a tame diagram of rank $i$ (p.19 [94]), we make two changes. First, in property (D2), we require that if 0 -squares $S_{1}, \ldots, S_{k}$ form a subdiagram $\mathrm{E}$ as in the definition of a 0 -bond and $p=q=\partial \Pi$, where $\Pi$ is a cell of rank $j>0$ in $\Delta$, then $\mathrm{E}$ is a 0 -bond between $\partial \Pi$ and $\partial \Pi$ in $\Delta$. Second, we add the following property. 
(D3) $\Delta$ contains no contractible $y$-annuli.

In the definition of a complete system (p.23 [94]) we require in (E3) that $e$ be a $y$-edge.

In Lemma 4.2 , the strict length $\left\|s_{1}\right\|,\left\|s_{2}\right\|$ of $s_{1}, s_{2}$ is meant.

In the definition of the weight function $\nu$ (p.28 [I94]), we require in (F1) that $e$ be a $y$-edge. In (F2), we allow that $e$ is not a $y$-edge.

In the beginning of the proof of Lemma 6.5, we note that the lemma is obvious if $\Delta$ contains no cells of positive rank. In general, repeating arguments of [194], we always understand "cells" as cells of positive rank and keep in mind the existence of cells of rank 0 .

In the definition of the height $h(W)$ of a word $W$ (p.89 [194]), we additionally set

$$
h(W)=\frac{1}{2}
$$

if $W \stackrel{i}{\neq} 1$ and $W$ is conjugate in $\operatorname{rank} i$ to a word $U$ with $|U|=0$ (that is, $U \in Q$ and $U \stackrel{i}{\neq} 1$ ).

In Lemma 10.2, we allow the extra case when $h(W)=\frac{1}{2}$.

Here is a new version of Lemma 10.4.

Lemma 10.4. (a) If a word $W$ has finite order $d>1$ in the group $\mathcal{G}(i)$ then $n^{3}$ is divisible by $d$.

(b) Every word $W$ with $|W| \leq i$ has finite order in rank $i$.

Proof. (a) By Lemma 10.2, either $h(W)=\frac{1}{2}$ (and then our claim is immediate from the construction of the group $Q$, see the definition of presentation (13)) or, otherwise, $W$ is conjugate in rank $i$ to a word of the form $A^{k} F$, where $A$ is a period of rank $j \leq i, 0<k<n^{3}$ and $F \in \mathcal{F}(A)$. In the latter case, it follows from Lemma 18.5(c) in rank $j-1<i$ that

$$
\left(A^{k} F\right)^{n^{2}} \stackrel{j-1}{=} A^{k n^{2}}
$$

Therefore,

$$
\left(A^{k} F\right)^{n^{3}} \stackrel{j-1}{=} A^{k n^{3}} \stackrel{j}{=} 1,
$$

whence $W^{n^{3}} \stackrel{i}{=} 1$ as required.

(b) By induction, it suffices to show that every word $W$ with $|W|=i$ has finite order in rank $i$ (for $i=0$ this is obvious). It follows from the definition of periods of rank $i \geq 1$ that if $W$ has infinite order in rank $i-1$ then $W^{n^{2}}$ is conjugate in rank $i-1$ to $A^{ \pm n^{2}}$, where $A$ is a period of rank $i$. Therefore, $W^{n^{3}} \stackrel{i}{=} 1$ as desired.

Lemma 10.4 is proved.

In Lemma 10.8, we drop part (b) of its conclusion (and keep in mind that the term "reducible cell" now means " $y$-annulus"). Note that the height of $\varphi\left(q_{1} t_{1}\right)$ in Lemma 10.8 is $m \geq 1$ hence noncontractible $y$-annuli in $\Delta_{0}, \Delta_{0}^{r}$ are impossible (for otherwise, the height $h\left(\varphi\left(q_{1} t_{1}\right)\right)$ of $\varphi\left(q_{1} t_{1}\right)$ would be at most $\left.\frac{1}{2}\right)$.

Lemma 10.9 is no longer needed for no path $q$ is (weakly) $j$-compatible with itself.

In the definition of a $U$-diagram of rank $i$ (p.134 [94]), we allow in property (U3) that the height $h(\varphi(e))$ of $\varphi(e)$ is $\frac{1}{2}$. 
Lemma 12.3 now claims that there are no $U$-diagrams of rank $i$. Recall that this agrees with our convention that if the conclusion states the existence of selfcompatible cells or $j$-compatibility of type (A2) then the assumption is false.

The analogues of Lemmas 13.1-16.6 are not needed.

In the hypothesis of Lemma 17.1, we now suppose that one can obtain from $\Delta_{0}$ an annular reduced diagram of rank $i$ which contains no noncontractible $y$-annuli by means of removal of reducible pairs and reducible $y$-annuli.

According to our convention, in the statement of Lemma 17.2, we replace the phrase "one has to remove a reducible cell to reduce $\Delta_{0}$ " by "one encounters a noncontractible $y$-annulus when reducing $\Delta_{0}$ ". In the conclusion of Lemma 17.2 and in its proof, we disregard reducible cells, $\mathcal{F}\left(A_{j}\right)$-involutions and consider, instead, noncontractible $y$-annuli and their 0-squares.

The new version of Lemma 17.3 is stated as follows.

Lemma 17.3. Let $\Delta$ be a disk reduced diagram of rank $i$ whose contour is $\partial \Delta=$ bpcq, where $\varphi(p)$ and $\varphi(q)^{-1}$ are A-periodic words and $A$ is a simple in rank $i$ word with $|A|=i+1$ (in particular, $A$ is a period of rank $i+1$ ). Suppose also that $\Delta$ itself is a contiguity subdiagram between $p$ and $q$ with $\min (|p|,|q|)>L|A|$. Then, in the notation of Lemmas 9.1-9.4, there exists a rigid subdiagram of the form $\Delta\left(m_{1}, m_{2}\right)$ in $\Delta(=\Delta(1, k))$ such that

$$
r\left(\Delta\left(m_{1}, m_{2}\right)\right)=0
$$

and the following analogues of inequalities (17.25)-(17.26) (p.222 [94) hold.

$$
\begin{aligned}
\left|q\left(m_{2}, m_{1}\right)\right| & >|q(k, 1)|-4.4|A|>(L-4.4)|A|, \\
\left|p\left(m_{1}, m_{2}\right)\right| & >|p(1, k)|-4.4|A|>(L-4.4)|A|, \\
\left|x_{m_{1}}\right| & =\left|y_{m_{2}}\right|=0 .
\end{aligned}
$$

Proof. To prove this new version of Lemma 17.3, we repeat the argument of the beginning of the proof of Lemma 17.3 [194]. As there, making use of Lemma 17.2, we prove Lemma 17.3.1. After that, arguing as in the proof of Lemma 17.3.2, it is easy to show, using Lemma 12.3 , that $r\left(\Delta\left(m_{1}, m_{2}\right)\right)=0$.

When proving the analogue of Lemma 18.2, we pick a word $B=B\left(a_{1}, a_{2}\right)$ in the alphabet $\left\{a_{1}, a_{2}\right\}$ of length $i+1$ so that $B$ has the same properties as those in I94 and, in addition, first and last letters of $B$ are distinct (the existence of such a word easily follows from Lemma 1.7 [194). Next, consider a word $B\left(a_{1}, a_{2}, q\right)$ in the alphabet $\left\{a_{1}, a_{2}, Q\right\}$ which is obtained from $B\left(a_{1}, a_{2}\right)$ by plugging in an element $q \in Q, q \neq 1$, between each pair of consecutive letters of the word $B\left(a_{1}, a_{2}\right)$. Then we replace each occurrence of the letter $a_{1}$ in $B\left(a_{1}, a_{2}, q\right)$ by $y$ and each occurrence of the letter $a_{2}$ in $B\left(a_{1}, a_{2}, q\right)$ by $y^{-1}$. Clearly, we have a word $B\left(y, y^{-1}, q\right)$ with $\left|B\left(y, y^{-1}, q\right)\right|=i+1$. Now, in view of Lemmas $10.2,10.4$, we can repeat the arguments of the proof of Lemma 18.2 without any changes.

Let $A$ be a period of rank $i+1$. By

denote a maximal subgroup of $Q \subseteq \mathcal{G}(i)$ (the fact that $Q$ naturally embeds in $\mathcal{G}(i)$ is immediate from Lemma 6.2) with respect to the property that $A$ normalizes this subgroup $\mathcal{F}(A) \subseteq \mathcal{G}(i)$.

Here is a new version of Lemma 18.3. 
Lemma 18.3. Suppose that $A$ is a period of rank $i+1$. Furthermore, let $\Delta$ be a disk reduced diagram of rank $i$ such that $\partial \Delta=b p c q$, where $\varphi(p), \varphi(q)^{-1}$ are $A$ periodic words with $\min (|p|,|q|)>1.1 n^{2}|A|$, and $\Delta$ itself be a contiguity subdiagram between sections $p$ and $q$. Then there exists a 0 -bond $\mathrm{E}$ in $\Delta$ with the standard contour $\partial \mathrm{E}=b_{\mathrm{E}} p_{\mathrm{E}} c_{\mathrm{E}} q_{\mathrm{E}}$, where $\left(p_{\mathrm{E}}\right)_{-},\left(q_{\mathrm{E}}\right)_{+}$are phase vertices of $p, q$, respectively, such that

$$
\varphi\left(b_{\mathrm{E}}\right) \in \mathcal{F}(A), \quad \varphi\left(b_{\mathrm{E}}\right) \in B(\mathcal{B} \cup c, n) \subset Q, \quad A^{n^{2}} \varphi\left(b_{\mathrm{E}}\right) A^{-n^{2}} \stackrel{i}{=} \varphi\left(b_{\mathrm{E}}\right)
$$

and for every integer $k$ one has

$$
\left(A^{k} \varphi\left(b_{\mathrm{E}}\right)\right)^{n^{2}} \stackrel{i}{=} A^{k n^{2}} .
$$

Proof. Lemma 17.3 enables us to assume that

$$
r(\Delta)=0, \quad \min (|p|,|q|)>\left(1.1 n^{2}-5\right)|A|, \quad|b|=|c|=0 .
$$

In particular, there are $|p|$ 0-bonds between $p$ and $q$ in $\Delta$. Let $\mathrm{E}$ be a 0 -bond between sections $p$ and $q$ and

$$
\partial \mathrm{E}=b_{\mathrm{E}} p_{\mathrm{E}} c_{\mathrm{E}} q_{\mathrm{E}}
$$

be the standard contour of $\mathrm{E}$, where $p_{\mathrm{E}}=\mathrm{E} \wedge p, q_{\mathrm{E}}=\mathrm{E} \wedge q$. It is clear that $\operatorname{div}\left(\left(p_{\mathrm{E}}\right)_{-},\left(q_{\mathrm{E}}\right)_{+}\right)$does not depend on E. Suppose that

$$
\operatorname{div}\left(\left(p_{\mathrm{E}}\right)_{-},\left(q_{\mathrm{E}}\right)_{+}\right) \neq 0 .
$$

$A$-Periodically extending $p$ or $q^{-1}$ on the left as in the beginning of the proof of Lemma 17.3 (see Fig. 17.4(a)-(b) in [I94]), we will get a diagram $\Delta^{\prime}$ with $\partial \Delta^{\prime}=b^{\prime} p^{\prime} c^{\prime} q^{\prime}$ such that both $\varphi\left(p^{\prime}\right)$ and $\varphi\left(q^{\prime}\right)^{-1}$ begin with a cyclic permutation $\bar{A}$ of $A$.

As in the proof of Lemma 17.1, we can easily get, making use of (15), that the annular diagram $\Delta_{0}^{\prime}$ (obtained from $\Delta^{\prime}$ as in Lemma 17.1) contains no $y$-annuli, in particular, $\Delta_{0}^{\prime}$ is already reduced. Therefore, Lemma 17.1 applies to $\Delta^{\prime}$ and yields that $\varphi\left(b^{\prime}\right) \stackrel{i}{=} 1$. It follows from (15) that $\left|b^{\prime}\right|>0$ and so $\bar{A}$ is not cyclically reduced in rank $i$. This contradiction proves that (15) is false and

$$
\operatorname{div}\left(\left(p_{\mathrm{E}}\right)_{-},\left(q_{\mathrm{E}}\right)_{+}\right)=0 .
$$

Without loss of generality, we may assume that words $\varphi(p), \varphi(q)^{-1}$ start with $A$ and the word $A$ starts with $y^{-1}$.

Using the notation of Lemma 9.1 , let $\mathrm{E}_{1}, \ldots, E_{|p|}$ be all (consecutive along $p$ ) 0 -bonds between $p$ and $q$ with standard contours

$$
\partial \mathrm{E}_{\ell}=b_{\ell} p_{\ell} c_{\ell} q_{\ell},
$$

where $p_{\ell}=\mathrm{E}_{\ell} \wedge p, q_{\ell}=\mathrm{E}_{\ell} \wedge q$ and $1 \leq \ell \leq|p|$.

Also, we let $|A|=m$ and consider words

$$
V_{0}=\varphi\left(b_{1}\right), V_{1}=\varphi\left(b_{m+1}\right), \ldots, V_{t}=\varphi\left(b_{m t+1}\right), \ldots, V_{n^{2}+1}=\varphi\left(b_{m\left(n^{2}+1\right)+1}\right)
$$

(recall that $\left.|p|>\left(1.1 n^{2}-5\right)|A|>\left(n^{2}+5\right)|A|\right)$. It is clear that

$$
A^{-1} V_{t} A=V_{t+1}
$$

and $V_{t}, V_{t+1}$ are words in $Q$, where $t=0, \ldots, n^{2}$.

Recall that the group $Q=P \lambda B(\mathcal{X} \cup c, n)$ is a semidirect product of the free Burnside group

$$
B(\mathcal{X}, n)=\left\langle x_{k}^{\omega_{0}} \mid k \in I\right\rangle
$$


and the direct product

$$
P=K \times B(\mathcal{B} \cup c, n)
$$

of $K=H / H^{n}=\left\langle a_{k}^{\gamma_{0}} \mid k \in I\right\rangle$ and the free Burnside group $B(\mathcal{B} \cup c, n)=$ $\left\langle c^{\delta_{0}}, b_{k}^{\delta_{0}} \mid k \in I\right\rangle$ so that the conjugation of $P$ by $x_{k}^{\omega_{0}}$ induces the automorphism (11).

Therefore, it follows from the definition of the group $\mathcal{G}=\mathcal{G}(0)$ given by (13) that every $V_{t}, t=0,1, \ldots, n^{2}$, belongs to the subgroup

$$
B(\mathcal{B} \cup c, n) \subset Q
$$

which is a factor of $P$ (otherwise, $y V_{t} y^{-1} \notin Q$, see (13)).

Consider the quotient of $\mathcal{G}$ by the normal closure $\left\langle\left\langle H / H^{n}\right\rangle\right\rangle$ of

$$
H / H^{n}=\left\langle a_{k}^{\gamma_{0}} \mid k \in I\right\rangle
$$

and let

be the natural homomorphism.

$$
\varphi_{0}: \mathcal{G} \rightarrow \mathcal{G} /\left\langle\left\langle H / H^{n}\right\rangle\right\rangle
$$

It is clear that $Q^{\varphi_{0}}$ is naturally isomorphic to $Q$, so we can assume that $Q^{\varphi_{0}}$ itself is a splitting extension of $B(\mathcal{B} \cup c, n)=B(\mathcal{B} \cup c, n)^{\varphi_{0}}$ by $B(\mathcal{X}, n)=B(\mathcal{X}, n)^{\varphi_{0}}$ so that

$$
x_{j}^{\omega_{0}} c^{\delta_{0}} x_{j}^{-\omega_{0}}=c^{\delta_{0}} b_{j}^{\delta_{0}}, \quad x_{j}^{\omega_{0}} b_{j^{\prime}}^{\delta_{0}} x_{j}^{-\omega_{0}}=b_{j^{\prime}}^{\delta_{0}}, j, j^{\prime} \in I .
$$

Observe that it follows from $V_{t} \in B(\mathcal{B} \cup c, n)$ that $V_{t}^{\varphi_{0}}=V_{t}$.

Consider the quotient $\mathcal{G}^{\varphi_{0}} /\left\langle\left\langle y^{\varphi_{0}}\right\rangle\right\rangle$ and let

$$
\varphi_{1}: \mathcal{G} \rightarrow \mathcal{G}^{\varphi_{0}} /\left\langle\left\langle y^{\varphi_{0}}\right\rangle\right\rangle
$$

denote the natural homomorphism. Clearly, $V_{t}^{\varphi_{1}}=V_{t}$, so

$$
V_{t+1}=A^{-\varphi_{1}} V_{t} A^{\varphi_{1}}, \quad t=0,1, \ldots, n^{2}-1,
$$

and hence

$$
V_{n^{2}}=\left(A^{-\varphi_{1}}\right)^{n^{2}} V_{0}\left(A^{\varphi_{1}}\right)^{n^{2}} .
$$

Since $A^{\varphi_{1}} \in Q^{\varphi_{1}}, Q^{\varphi_{0}}$ and $Q^{\varphi_{1}}$ are naturally isomorphic and $Q^{\varphi_{0}}$ has exponent $n^{2}$, it follows that $V_{n^{2}}=V_{0}$. Now it is obvious that $V_{0} \in \mathcal{F}(A)$ and

$$
A^{n^{2}} V_{0} A^{-n^{2}} \stackrel{i}{=} V_{n^{2}} \stackrel{i}{=} V_{0} .
$$

At last, we have

$$
\begin{aligned}
\left(A^{k} V_{0}\right)^{n^{2}} \stackrel{0}{=} A^{k} V_{0} A^{-k} A^{2 k} V_{0} A^{-2 k} \ldots A^{k n^{2}} V_{0} A^{-k n^{2}} A^{k n^{2}} \stackrel{i}{=} & \\
\left(A^{\varphi_{1}}\right)^{k} V_{0}\left(A^{\varphi_{1}}\right)^{-k}\left(A^{\varphi_{1}}\right)^{2 k} V_{0}\left(A^{\varphi_{1}}\right)^{-2 k} & \ldots\left(A^{\varphi_{1}}\right)^{k n^{2}} V_{0}\left(A^{\varphi_{1}}\right)^{-k n^{2}} A^{k n^{2}} \stackrel{0}{=} \\
& \left(\left(A^{\varphi_{1}}\right)^{k} V_{0}\right)^{n^{2}}\left(A^{\varphi_{1}}\right)^{-k n^{2}} A^{k n^{2}} \stackrel{i}{=} A^{k n^{2}}
\end{aligned}
$$

and Lemma 18.3 is proven.

Lemma 18.4 is not needed.

Let us state a new version of Lemma 18.5.

Lemma 18.5. Let $A$ be a period of rank $i+1$ and $\mathcal{F}(A)$ be a maximal subgroup of $Q \subset \mathcal{G}(i)$ with respect to the property that $A$ normalizes $\mathcal{F}(A)$. Then the following are true.

(a) The subgroup $\mathcal{F}(A)$ is defined uniquely.

(b) Suppose $\Delta$ is a disk reduced diagram of rank $i$ with $\partial \Delta=b p c q$, where $\varphi(p)$, $\varphi(q)^{-1}$ are A-periodic words with $\min (|p|,|q|)>\beta n^{3}|A|$, such that $\Delta$ itself is a 
contiguity subdiagram between $p$ and $q$. Then there is a 0-bond $\mathrm{E}$ between $p$ and $q$ with the standard contour $\partial \mathrm{E}=b_{\mathrm{E}} p_{\mathrm{E}} c_{\mathrm{E}} q_{\mathrm{E}}$, where $p_{\mathrm{E}}=\mathrm{E} \wedge p, q_{\mathrm{E}}=\mathrm{E} \wedge q$, such that $\left(p_{\mathrm{E}}\right)_{-},\left(q_{\mathrm{E}}\right)_{+}$are phase vertices of $p, q$, respectively, and $\varphi\left(b_{\mathrm{E}}\right) \in \mathcal{F}(A)$.

(c) $A^{n^{2}}$ centralizes the subgroup $\mathcal{F}(A)$ and $\mathcal{F}(A)$ has exponent $n$. Furthermore, if $F \in \mathcal{F}(A)$ and $k$ is an integer then

$$
\left(A^{k} F\right)^{n^{2}} \stackrel{i}{=} A^{k n^{2}} .
$$

(d) The subgroup $\langle\mathcal{F}(A), A\rangle$ of $\mathcal{G}(i)$ has the property that a word $X$ belongs to $\langle\mathcal{F}(A), A\rangle$ if and only if there is an integer $m \neq 0$ such that $X A^{m} X^{-1} \stackrel{i}{=} A^{m}$.

Proof. (a) This is obvious from definitions.

(b) Since $\beta n^{3}|A|>1.1 n^{2}|A|$, we can apply Lemma 18.3 to $\Delta\left(m_{1}, m_{2}\right)$ which yields the required conclusion.

(c) Suppose that $X \in \mathcal{F}(A)$. Picking sufficiently large $\ell$, we have an equality

$$
A^{-\ell} X A^{\ell} \stackrel{i}{=} Y,
$$

where $Y \in \mathcal{F}(A)$. Consider a reduced diagram $\Delta$ of rank $i$ for this equality. Using Lemmas $6.5,3.1$ and inequality $\ell \gg 1$, it is easy to show that $\Delta$ contains a contiguity subdiagram $\Delta_{0}$ between sections whose labels are $A^{-\ell}$ and $A^{\ell}$ with the standard contour

$$
\partial \Delta_{0}=b p c q,
$$

where $\varphi(p), \varphi(q)^{-1}$ are $A$-periodic sections longer than $\beta n^{3}|A|$. Now we can refer to Lemma 18.3 and conclude that $X$ belongs to the double coset

$$
\langle A\rangle \varphi\left(b_{\mathrm{E}}\right)\langle A\rangle \subseteq \mathcal{G}(i),
$$

where $\varphi\left(b_{\mathrm{E}}\right)$ is from the conclusion of Lemma 18.3. Since $X \in Q$ has finite order, it follows from Lemma 18.3 that $X \stackrel{i}{=} A^{\ell^{\prime}} \varphi\left(b_{\mathrm{E}}\right) A^{-\ell^{\prime}}$ with some $\ell^{\prime}$. Therefore, $X$ commutes with $A^{n^{2}}$ in rank $i$ just like $\varphi\left(b_{\mathrm{E}}\right)$ does and, by Lemma 18.3,

$$
X^{n} \stackrel{i}{=} 1, \quad\left(A^{k} X\right)^{n^{2}} \stackrel{i}{=} A^{k n^{2}} .
$$

Part (c) is proven.

(d) By part (c), it suffices to show that an equality

$$
X A^{m} X^{-1} \stackrel{i}{=} A^{m},
$$

where $m \neq 0$, implies that $X \in\langle A, \mathcal{F}(A)\rangle$. Arguing exactly as in the proof of part (c), we can show that

$$
X \in\langle A\rangle \varphi\left(b_{\mathrm{E}}\right)\langle A\rangle \subseteq \mathcal{G}(i),
$$

where $\varphi\left(b_{\mathrm{E}}\right) \in \mathcal{F}(A)$ by Lemma 18.3. Thus $X \in \mathcal{F}(A)$ and Lemma 18.5 is proven.

Here is a new version of Lemma 19.1.

Lemma 19.1. There is no disk diagram $\Delta$ of rank $i$ such that $\partial \Delta=b p c q$, where $p, q$ are A-periodic sections with $|p|,|q|>\frac{1}{3} \beta n^{3}|A|, A$ is a period of rank $i+1$, and $\Delta$ itself is a contiguity subdiagram between $p$ and $q$.

Proof. Arguing on the contrary, we assume the existence of such a diagram $\Delta$ and, replacing the coefficient $N\left(N\right.$ is defined in (17.1) on p.212 [94]) by $\frac{1}{3} \beta n^{3}$, 
repeat the proof of Lemma 19.1 [94 up to getting equality (19.23) (p.290 [94) which now reads

$$
\varphi(d) A^{n^{2}} \varphi(d)^{-1} \stackrel{i}{=} A^{-n^{2}} .
$$

Lemma 19.1.3. The equality (16) is impossible.

Proof. Arguing on the contrary, we note that it follows from (16) that

$$
\varphi(d)^{2} A^{n^{2}} \varphi(d)^{-2} \stackrel{i}{=} A^{n^{2}} .
$$

Recall that $|A|=i+1$ and, by Lemma 19.1.1, $\varphi(d)$ is conjugate in rank $i$ to a word $W$ with

$$
|W|<(0.5+\xi)|A|<|A| \text {. }
$$

Hence, by Lemma 10.4(b), $\varphi(d)$ has finite order in rank $i$ and, by Lemma 10.4(a),

$$
\varphi(d)^{n^{3}} \stackrel{i}{=} 1 \text {. }
$$

This means that $\varphi(d) \in\left\langle\varphi(d)^{2}\right\rangle$ in rank $i$ and so equalities (16)-(17) imply that

$$
A^{2 n^{2}} \stackrel{i}{=} 1 \text {. }
$$

A contradiction to the definition of a period of rank $i+1$ completes proofs of Lemmas 19.1.3 and 19.1.

Analogues of Lemmas 19.2-19.6 are no longer needed.

The statements and proofs of Lemmas 20.1-20.2 are retained.

Having made all necessary changes, we can now turn to the group $\mathcal{G}(\infty)$ given by the presentation (14).

It follows from Lemma 10.4(b) that every word $W$ has finite order in rank $i \geq$ $|W|$. Then, by Lemma 10.4(a),

$$
W^{n^{3}} \stackrel{i}{=} 1
$$

provided that $i \geq|W|$. Thus, the group $\mathcal{G}(\infty)$ has exponent $n^{3}$.

Suppose that $W$ is a word with $|W|=0$, that is, $W \in Q$. Let $W=1$ in the group $\mathcal{G}(\infty)$. Then there is an $i$ such that $W \stackrel{i}{=} 1$. Consider a reduced diagram $\Delta$ for this equality. Since $|\partial \Delta|=0$, it follows from Lemma 6.2 that $r(\Delta)=0$, that is, $\varphi(\partial \Delta)=1$ in $Q$. Thus $Q$ naturally embeds in $\mathcal{G}(\infty)$.

Lemmas B and $\mathrm{C}$ are proven.

\section{Proofs of Theorems A And B}

We start with proving Theorem B. Let $N_{0}>2^{16}$ be odd and

$$
G_{0}=\left\langle\mathcal{A}_{0} \| R=1, R \in \mathcal{R}_{0}\right\rangle
$$

be a finite presentation of a group $G_{0}$ with $\left|\mathcal{A}_{0}\right|>1$.

Applying the construction of presentation (10), we set $\mathcal{A}=\mathcal{A}_{0}, \mathcal{R}=\mathcal{R}_{0}, H=$ $G_{0}, n=N_{0}$. Denote the corresponding sets of generators and relators of thus constructed presentation (10) by $\mathcal{A}_{1}, \mathcal{R}_{1}$. The group given by (10) denote by $G_{1}$. Since $\mathcal{A}_{0} \subset \mathcal{A}_{1}, \mathcal{R}_{0} \subset \mathcal{R}_{1}$, we can speak of the subgroup $\left\langle\mathcal{A}_{0}\right\rangle$ of $G_{1}$ generated by the set $\mathcal{A}_{0}$. It follows from Lemma A that the subgroup $\left\langle\mathcal{A}_{0}\right\rangle \subseteq G_{1}$ is naturally isomorphic to the quotient $G_{0} / G_{0}^{N_{0}}$.

At the second step, we again apply the construction of (10) with $\mathcal{A}=\mathcal{A}_{1}$, $\mathcal{R}=\mathcal{R}_{1}, H=G_{1}$, and $n=N_{1}=N_{0}^{3}$. Denote the corresponding sets of generators and relators of thus constructed presentation (10) by $\mathcal{A}_{2}, \mathcal{R}_{2}$ and denote the group 
given by (10) by $G_{2}$. It follows from Lemmas A-B that the subgroups $\left\langle\mathcal{A}_{0}\right\rangle,\left\langle\mathcal{A}_{1}\right\rangle$ of $G_{2}$ are naturally isomorphic to $G_{0} / G_{0}^{N_{0}}, G_{1} / G_{1}^{N_{1}}$, respectively, and $G_{0} / G_{0}^{N_{0}}$ naturally embeds in $G_{1} / G_{1}^{N_{1}}$.

Keeping on doing this, we will inductively define a finite presentation

$$
G_{\ell+1}=\left\langle\mathcal{A}_{\ell+1} \| R=1, R \in \mathcal{R}_{\ell+1}\right\rangle, \quad \ell \geq 1,
$$

which is constructed as presentation (10), where $\mathcal{A}=\mathcal{A}_{\ell}, \mathcal{R}=\mathcal{R}_{\ell}, H=G_{\ell}$, and

$$
n=N_{\ell}=N_{\ell-1}^{3}=N_{0}^{3^{\ell}} .
$$

It follows from Lemmas A-B that the subgroups $\left\langle\mathcal{A}_{\ell-1}\right\rangle,\left\langle\mathcal{A}_{\ell}\right\rangle$ of $G_{\ell+1}$ are naturally isomorphic to

$$
G_{\ell-1} / G_{\ell-1}^{N_{\ell-1}} \subseteq G_{\ell} / G_{\ell}^{N_{\ell}}, \quad G_{\ell} / G_{\ell}^{N_{\ell}},
$$

respectively. Therefore, by induction, the subgroup $\left\langle\mathcal{A}_{k}\right\rangle$ of $G_{\ell+1}$, where $0 \leq k \leq \ell$, is naturally isomorphic to $G_{k} / G_{k}^{N_{0}^{3 k}}$.

Now define

$$
G_{\infty}=\left\langle\mathcal{A}_{\infty}=\bigcup_{j=0}^{\infty} \mathcal{A}_{j} \| R=1, R \in \mathcal{R}_{\infty}=\bigcup_{j=0}^{\infty} \mathcal{R}_{j}\right\rangle
$$

It is clear that for every $\ell \geq 0$ the subgroup $\left\langle\mathcal{A}_{\ell}\right\rangle$ of $G_{\infty}$ is naturally isomorphic to $G_{\ell} / G_{\ell}^{N_{\ell}}$ and that if $W_{1}, \ldots, W_{m}$ are words in $\mathcal{A}_{\ell}^{ \pm 1}$ then they generate naturally isomorphic subgroups in $G_{\infty}$ and in $G_{\ell+1}$. Note that it is immediate from the construction of the group $\mathcal{G}(\infty)$ (see (14) in which we put $\mathcal{A}=\mathcal{A}_{\ell-1}, \mathcal{R}=\mathcal{R}_{\ell-1}$, $H=G_{\ell-1}, n=N_{0}^{3^{\ell-1}}$ ) and Lemma $\mathrm{C}$ that $G_{\ell} / G_{\ell}^{N_{0}^{3^{\ell}}}$ is not a locally finite group when $\ell \geq 1$. The proof of Theorem B is complete.

Theorem A is immediate from Theorem B if we pick a free group of rank 2 as $G_{0}$ (then $G_{0} / G_{0}^{N_{0}}$ is a free 2-generator Burnside group $B\left(2, N_{0}\right)$ of exponent $N_{0}$ ).

\section{REFERENCES}

[siA75] S.I. Adian, The Burnside problem and identities in groups, Nauka, Moscow, 1975; English translation: Springer-Verlag, 1979.

[svA72] S.V. Aleshin, Finite automata and the Burnside problem for periodic groups, Math. Notes 11(1972), 199-203.

[B02] W. Burnside, On unsettled question in the theory of discontinuous groups, Quart. J. Pure and Appl. Math. 33(1902), 230-238.

[eG64] E.S. Golod, On nil-algebras and residually finite p-groups, Math. USSR Izvestiya 28(1964), 273-276.

[GS83] N. Gupta and S. Sidki, On the Burnside problem for periodic groups, Math. Z. 182(1983), 385-388.

[nG89] N. Gupta, On groups in which every element has finite order, Amer. Monthly 96(1989), 297-308.

[rG80] R.I. Grigorchuk, On the Burnside problem for periodic groups, Funcional Anal. Appl. 14(1980), 41-43.

[194] S.V. Ivanov, The free Burnside groups of sufficiently large exponents, Internat. J. Algebra and Comp. 4(1994), 1-308.

[I00] S.V. Ivanov, On finitely presented groups given by periodic relators, J. Group Theory 3(2000), 95-99.

[IO96] S.V. Ivanov and A.Yu. Ol'shanskii, Hyperbolic groups and their quotients of bounded exponents, Trans. Amer. Math. Soc. 348(1996), 2091-2138.

[IO97] S.V. Ivanov and A.Yu. Ol'shanskii, On finite and locally finite subgroups of free Burnside groups of large even exponents, J. Algebra 195(1997), 241-284. 
[L96] I.G. Lysenok, Infinite Burnside groups of even period, Math. Ross. Izvestiya 60(1996), 3-224.

[NA68] P.S. Novikov and S.I. Adian, On infinite periodic groups, I, II, III, Math. USSR Izvestiya 32(1968), 212-244, 251-524, 709-731.

[O82] A.Yu. Ol'shanskii, On the Novikov-Adian theorem, Math. USSR Sbornik 118(1982), 203-235.

[O89] A.Yu. Ol'shanskii, Geometry of Defining Relations in Groups, Nauka, Moscow, 1989; English translation in Math. and Its Applications (Soviet series) $\mathbf{7 0}$ (Kluwer Acad. Publishers, 1991).

Department of Mathematics, University of Illinois, Urbana, IL 61801

E-mail address: ivanov@math.uiuc.edu 